\title{
El aprendizaje servicio como metodología para la enseñanza de la Historia y el patrimonio local
}

\author{
Doi: https://doi.org/ 10.25100/hye.v15i53.8661 \\ Artículo recibido: 19-02-2018 Artículo aceptado: 31-07-2019
}

\section{Raimundo A. Rodríguez Pérez}

Es Licenciado en Historia y Doctor Europeo en Historia por la Universidad de Murcia. Cuenta con dos Máster en: Recursos Histórico-Culturales e Historia Comparada de las Relaciones Sociales y Familiares (con Mención de Calidad del MEC). Es Profesor Titular de Didáctica de las Ciencias Sociales en la Facultad de Educación de la Universidad de Murcia. Autor de 50 publicaciones sobre Historia Moderna y Didáctica de las Ciencias Sociales, así como de 25 ponencias y comunicaciones a congresos y seminarios internacionales. Sus actuales líneas de estudio son la Didáctica de la Historia en Educación Primaria y Secundaria, y la formación docente.

Departamento de Didáctica de las Ciencias Matemáticas y Sociales, Facultad de Educación, Campus de Espinardo, Universidad de Murcia, España.

Correo electrónico: raimundorodriguez@um.es

ORCID: 0000-0002-9634-569X

\section{Marta Gutiérrez Sánchez}

Es Licenciada en Pedagogía y Doctora en Pedagogía por la Universidad de Murcia. Cuenta con dos Diplomas en Magisterio y Estudios Avanzados por parte de la Universidad de Murcia. Profesora Es ayudante Doctora de Teoría de la Educación, imparte docencia en los Grados de Educación Social y Pedagogía, y en el Máster Inclusión-Exclusión Social y Educativa: Políticas, Programas y Prácticas.

Departamento de Teoría e Historia de la Educación. Facultad de Educación, Campus de Espinardo, Universidad de Murcia, España.

Correo electrónico: martags@um.es

ORCID: 0000-0001-7302-9283

Forma de citar este artículo: Rodríguez Pérez, A. Raimundo y Marta Gutiérrez Sánchez “El aprendizaje servicio como metodología para la enseñanza de la Historia y el patrimonio local". Historia y Espacio, vol. 15 n 53 (2019): 191-220. Doi.org/ 10.25100/hye.v15i53.8661.

Artículo Tipo 1: de investigación 
El aprendizaje servicio como metodología para la enseñanza de la Historia y el patrimonio local

Raimundo A. Rodríguez Pérez - Marta Gutiérrez Sánchez

\section{El aprendizaje servicio como metodología para la enseñanza de la Historia y el patrimonio local}

Resumen: El aprendizaje servicio es una metodología innovadora que comienza a tener relevancia. Consiste en formar a estudiantes en valores cívicos, que sean capaces de poner en práctica sus destrezas para afrontar problemas sociales. En el ámbito de la enseñanza de la Historia y el patrimonio, puede devenir en una metodología alternativa, englobada en las estrategias de indagación y técnicas cooperativas, en la que los estudiantes tengan un papel activo. Sin embargo, este tipo de métodos, con ser harto interesantes, no suelen redundar en algo útil para la sociedad. El aprendizaje servicio puede suplir algunas carencias en los modelos formativos que vienen utilizándose, al aportar una colaboración activa entre entidades educativas y colectivos sociales que se ocupan de problemas que afectan al contexto donde vive el alumnado.

Así pues, se propone que, para una mejor enseñanza de la historia en niveles educativos iniciales, estudien los problemas de conservación del patrimonio de su entorno próximo y el paisaje en que se ubica. En este texto se hace una propuesta para que los estudiantes del grado en educación primaria de la Universidad de Murcia (España) analicen la historia y el patrimonio local, colaborando con asociaciones cívicas que promueven la conservación de un patrimonio que suelen desconocer.

Palabras clave: Aprendizaje servicio, Enseñanza de la Historia, Educación primaria, Historia y patrimonio local, Maestros en formación.

\section{Service-learning as a methodology for teaching of local history and heritage}

Abstract: Service-learning is an innovative methodology that begins to have relevance. It consists of training students in civic values, who are able to put their skills to face social problems. In the field of history and heritage teaching it can become an alternative methodology, encompassed in inquiry strategies and cooperative techniques, where students play an active role. However this type of methods with being very interesting do not usually result in something useful for society. Service-learning can fill some gaps in the training models that are being used by providing active collaboration between educational entities and social groups, which deal with problems that affect the context in which students live. It is proposed that, for a better teaching of history at initial educational levels, study the problems of conservation of the heritage of their immediate environment and the landscape in which it is located. In this text a proposal is made so that the students of the Degree in Primary Education of the University of Murcia (Spain) analyze local history and heritage, collaborating with civic associations that promote the conservation of a heritage that they usually do not know.

Keywords: Service-learning, Teaching history, Primary education, Local history and heritage, Teachers in training. 


\section{Raimundo A. Rodríguez Pérez-Marta Gutiérrez Sánchez}

\section{El aprendizaje servicio como metodología para la enseñanza de la Historia y el patrimonio local}

\section{Introducción}

El aprendizaje servicio ${ }^{1}$ es una metodología que comienza a tener relevancia en el ámbito educativo pues posee un cúmulo de características innovadoras. Así, sobresale la formación de estudiantes en valores cívicos para que sean capaces de poner en práctica todas sus habilidades sociales al momento de afrontar los problemas de su entorno, desde la reciprocidad de perspectivas. También destaca la enseñanza de la Historia como pasado potencia, porque pone en valor "los contextos sociales" y engloba estrategias de indagación, reflexión y cooperación, involucrando a los estudiantes desde un papel activo y trascendiendo la historia como una sucesión de hechos aislados. Por su parte, busca la colaboración activa entre entidades educativas y colectivos sociales que se ocupan de problemas que afectan al contexto en que vive el alumnado e implica nuevas perspectivas respecto a estrategias como las salidas escolares y los proyectos de aprendizaje basado en problemas o estudio de casos; instancias que, siendo interesantes, no logran transformar la sociedad receptora.

A partir de lo descrito, se activa la práctica de una metodología que transita la participación, profundizando la ciudadanía en la escuela, es decir, el acceso a la educación y a la información propendiendo a la inserción al mundo del trabajo con experiencia adquirida; poniendo en valor una mejor enseñanza de la Historia en niveles educativos iniciales que re conceptualicé los problemas de conservación del patrimonio en su entorno próximo y el paisaje en que se ubica. En síntesis, se trata de una propuesta analítica y contextualizada en la que los estudiantes del grado en educación primaria de la Universidad de Murcia trabajarán conjuntamente con colectivos sociales que promueven la defensa y conservación de un patrimonio en peligro en la ciudad española de Murcia

1 Este trabajo forma parte del Proyecto de Investigación 20638/JLI/18 “Competencias docentes y métodos activos de aprendizaje. Una investigación evaluativa con el profesorado en formación de ciencias sociales", financiado por la Fundación Séneca. Agencia de Ciencia y Tecnología de la Región de Murcia (España)”. 
y su comarca ${ }^{2}$, por medio de la colaboración con entidades como Huermur, Huerta Viva y Legado.

La propuesta que se presenta queda justificada por algunas cuestiones que caracterizan nuestro contexto actual. El modelo económico actual se manifiesta a escala transnacional ejerciendo un permanente despojo sobre la naturaleza, las personas, su bagaje cultural y su entorno próximo mediante una dinámica de acumulación de bienes y expansión territorial constante en términos productivos y geopolíticos, tendiendo a la hegemonía global ${ }^{3}$.

En este escenario, se desconocen la historia y la cultura de una sociedad, excluyendo a las personas de los procesos políticos anteriores, separándolos de sus medios de producción y reproducción de la vida. El objetivo es reconvertir a las personas en fuerza de trabajo competitiva, libre, desposeída y, por ende, susceptible de ser explotada; transformando la naturaleza (en sentido amplio) en mero medio de producción y, a todos los procesos vivos que le son inherentes, en potenciales mercancías, implosionando relaciones sociales, tradiciones culturales y patrimonios históricos. Esta implosión se lleva a cabo a través de una doble operatoria invisibilización-hipervisibilización: se invisibiliza cualquier manifestación político-cultural cosmocéntrica que se organice a través de vínculos armónicos y de respeto mutuo con la naturaleza, en el marco de una totalidad compleja e integrada, en la que todos sus componentes interactúan como sujetos que se complementan y resuelven sus necesidades de manera recíproca, al tiempo que se hipervisibiliza un modelo antropocéntrico guido por una lógica egoísta, racional y maximizadora que tiene por objeto la renta, el prestigio y el poder de lograr la mayor cantidad de utilidades-beneficios individuales al menor costo posible.

Por otro lado, el pragmatismo y el utilitarismo parecen estar presentes en las relaciones sociales y, por tanto, las relaciones de tipo solidario parecen escasear entre los miembros de nuestra sociedad ${ }^{4}$. Este avasallamiento implica que parte de la población viva bajo la línea de la pobreza. Se reduce la realidad

2 María Manzanera, Murcia huertana (Murcia: Fundación Cajamurcia, 2014).

3 Claudia Composto y Mina Lorena Navarro, "Estados, transnacionales extractivas y comunidades movilizadas: dominación y resistencias en torno de la minería a gran escala en América Latina”, Revista Theomai 25, 1 (2012): 58-78.

4 Jordi Maiso, “Sobre la producción y reproducción social de la frialdad”, en Las víctimas como precio necesario, eds. José Antonio Zamora, Manuel Reyes Mate y Jordi Maiso (Madrid: Trotta, 2016), 51-69. 
a números que invisibilizan expectativas, sentimientos, sufrimientos y dolores de las personas.

Por su parte, el avance de la tecnología ha logrado la convergencia entre diferentes sistemas de comunicación (periódicos/radio/tv, Internet/redes sociales/dispositivos móviles) conformando relaciones fugaces de asociación entre las personas que propenden a la ruptura de lazos comunitarios y solidarios entre sus miembros. A su vez, la interconexión digital total y la comunicación en tiempo real dificultan cada vez más el encuentro con otros; sirviendo para encontrar personas con iguales gustos y pensamientos, obturando lo desconocido y distinto, segregando nuestro horizonte de experiencias; exaltando los aspectos más narcisistas de las personas que, a decir de ByunChul $^{5}$, hipervisibiliza una matriz de pensamiento autopropagandista que refuerza nuestras propias nociones, al tiempo que excluye todo el resto. Y, como afirma Bauman ${ }^{6}$, abundan en nuestra sociedad relaciones sociales frágiles, artificiales y líquidas que constituye una experiencia en sentido enfático de la negatividad de lo distinto y de la transformación. La negatividad de lo distinto da forma y medida a una mismidad que tiene una conformación, un recogimiento interior, una intimidad que se deben a la diferencia con lo distinto.

En este contexto, es necesario avanzar hacia una sociedad comprometida con la construcción de una sociedad más justa y sostenible. Se trata de avanzar hacia una ciudadanía crítica que se aleje de los valores que orquesta la sociedad de consumo, y de esa "ética light", acuñada por Torío", caracterizada por la carencia de compromisos y deberes por parte de la ciudadanía.

Así las cosas, este panorama obliga a pensar la formación universitaria en otros términos metodológicos. Pensar una propuesta pedagógica en términos más cívicos implica un replanteamiento gnoseológico en torno a la idea del bien común ${ }^{8}$ y un trabajo sobre presupuestos epistemológicos asentados en la solidaridad y la cooperación que habiliten una metodología de la concienciación y la sensibilización del alumnado con su entorno social para lograr la puesta en práctica de técnicas concretas que los relacionen de manera directa con aquellos contextos en los que desarrollarán su trabajo profesional.

5 Han Byun-Chul, La expulsión de lo distinto (Barcelona: Herder, 2017).

6 Zygmunt Bauman, Vida líquida (Barcelona: Paidós, 2006).

7 Susana Torío, "Familia, escuela y sociedad", Aula Abierta, 83 (2004): 35-52.

8 François Houtart, "De los bienes comunes al bien común de la humanidad”, El Ágora USB 14,1 (2014): 259-293. 
Es necesario que esta propuesta pedagógica no desconozca la matriz individualista y competitiva del mercado de trabajo, pero que logre trascenderla redefiniendo el núcleo central que articula la formación universitaria como una elaboración social en la que el pensamiento tiene un lugar esencial, pero también la experiencia concreta, en particular las luchas sociales, que corresponden cada una a una deficiencia de cumplimiento del bien común de la humanidad y que buscan soluciones. Dado que la globalización destructiva del capitalismo ha hegemonizado las economías, las sociedades y las culturas del mundo entero, pero no las ha eliminado completamente, la tarea es de todas las personas, según sus características y sus experiencias históricas. Para ello, es menester, durante la formación de grado, la adquisición de una serie de principios éticos y morales que pongan en valor dinámicas sociales equilibradas entre personas, géneros y grupos sociales, en armonía con la naturaleza, para promover la vida y asegurar su reproducción. Se trata de respetar la naturaleza en sentido amplio, es decir, de una perspectiva medioambiental en armonía con el ecosistema, poniendo en valor la historia y el patrimonio cultural de una sociedad. En palabras de Houtart ${ }^{9}$, se trata de vivir bien, de cumplir con el bien común de la humanidad; lo que implica, como primer paso, el respeto de la integridad de la naturaleza, como fuente de vida de la madre tierra.

\section{El aprendizaje servicio: concepto y virtualidades}

Se entiende el aprendizaje servicio como una propuesta pedagógica que combina la participación ciudadana con el aprendizaje de contenidos académicos. Requiere movilizar aprendizajes adquiridos dándoles sentido en una acción de servicio, por lo que es una metodología que promueve el aprendizaje crítico, reflexivo ${ }^{10}$ y activo. Asimismo, facilita la construcción de una ciudadanía activa y comprometida a través de acciones que tratan de mejorar y satisfacer las necesidades del entorno ${ }^{11}$. Se centra, por tanto, en dos núcleos fundamentales: el aprendizaje, situado dentro de pedagogías activas

9 Houtart, "De los bienes comunes", 263-265.

${ }^{10}$ Lauren Weiner Vaknin y Marilee J. Bresciani, “Implementing Quality Service-Learning Programs in Community Colleges”, Journal of Research and Practice 37, 12 (2013): 979-989, Doi: 10.108 0/10668926.2010.515515

${ }^{11}$ Miquel Martínez, "Aprendizaje servicio y construcción de ciudadanía activa en la universidad: la dimensión social y cívica de los aprendizajes académicos”, en Aprendizaje servicio y responsabilidad social de las universidades, ed. Miquel Martínez (Barcelona: Octaedro, 2008), 11-26. 
y en conexión con el entorno comunitario con el objetivo de mejorarlo, y el servicio, vinculado a prácticas de intervención ciudadana.

En un trabajado financiado por el Ministerio de Educación de Argentina ${ }^{12}$, se revisaron diferentes definiciones de aprendizaje servicio en diversas lenguas, constatándose que todas ellas presentan unos rasgos comunes, a saber:

1. Las acciones de aprendizaje servicio implican atender necesidades de la comunidad. Esto no sólo supone hacer un diagnóstico de la realidad, sino orquestar un conjunto de acciones que contribuyan a transformarla. Asimismo, es de vital importancia hacer partícipes del diagnóstico y de las acciones transformadoras a los individuos y comunidades que están afectados por la problemática que se pretende solucionar.

2. Las acciones de aprendizaje servicio implican dar un papel protagonista a los estudiantes. Los estudiantes han de involucrarse en todo el proceso. Han de asumir un papel activo desde la identificación de la necesidad o problema hasta el diseño y evaluación de la intervención que se pretende llevar a cabo.

3. Las acciones de aprendizaje servicio están íntimamente relacionadas con los contenidos de aprendizaje, lo que supone identificar aquellos elementos del currículum que se va a contribuir a desarrollar mediante la acción solidaria.

Los beneficios que aporta la participación en los proyectos de aprendizaje servicio son diversos, y se señalan en la literatura especializada, tanto del contexto español como de otros contextos, resultados muy positivos que redundan en la calidad de la formación de los estudiantes universitarios. En el ámbito anglosajón, Furco ${ }^{13}$ ha destacado resultados de carácter académico, cívico, moral, personal y social arguyendo que los participantes en experiencias de aprendizaje servicio desarrollan conocimientos y habilidades, comprenden mejor las cuestiones sociales, mejoran la habilidad para tomar decisiones independientes respecto a cuestiones morales y optimizan competencias para

\footnotetext{
${ }^{12}$ Ministerio de Educación de Argentina, Itinerario y herramientas para desarrollar un proyecto de aprendizaje-servicio (Buenos Aires: Ministerio de Educación de la Nación, 2012).

${ }^{13}$ Andrew Furco, "Impacto de los proyectos de aprendizaje-servicio", en Actas del séptimo Seminario Internacional Aprendizaje y Servicio Solidario (Buenos Aires, 2004), 19-26.
} 
el liderazgo y el trabajo en equipo. Por su parte, Glazier, Able y Charpentier ${ }^{14} \mathrm{y}$ Lockeman y Pelco ${ }^{15}$ han manifestado que este tipo de metodología contribuye a una educación de calidad y a una mejor contextualización de los aprendizajes adquiridos en la formación universitaria.

En el ámbito español, se ha constatado una mejora en el rendimiento académico. Ejemplo de ello es el trabajo realizado por Mella-Núñez, SantosRego y Malheiro-Gutiérrez ${ }^{16}$, quienes han argumentado que la participación del alumnado en proyectos de aprendizaje servicio produce un efecto positivo en una serie de factores mediadores (autoestima, empoderamiento, conducta prosocial, motivación y compromiso) que repercuten de manera positiva en el rendimiento académico.

Teniendo en cuenta lo expuesto, el aprendizaje servicio, más que erigirse como una estrategia metodológica, es un enfoque pedagógico integral porque, al combinar el aprendizaje académico con la participación en la comunidad, permite dotar de significatividad los contenidos que articulan los planes formativos, al tiempo que contribuye a desarrollar un pensamiento crítico y ético del entorno en que vivimos. La implicación del alumnado en la comunidad, identificando necesidades e interviniendo para mejorar la realidad, contribuye a tomar conciencia de situaciones de injusticia, cuestión fundamental si queremos avanzar hacia una sociedad más democrática y equitativa. Desde esta óptica, no se entiende la participación comunitaria que posibilita el aprendizaje servicio desde una perspectiva caritativa orientada a satisfacer las necesidades de individuos y comunidades, sino desde un marco que posibilita a los estudiantes tomar conciencia de los derechos sociales que el Estado tiene la obligación de garantizar.

En definitiva, el aprendizaje servicio es algo más que una metodología docente innovadora al permitir una educación ciudadana que atiende a las necesidades formativas de la sociedad actual. Mediante un correcto planteamiento, puede ser de gran utilidad para la enseñanza de la Historia y el patrimonio local, dado que moviliza una serie de valores y actitudes de gran

${ }^{14}$ Jocelyn Glazier, Harriet Able y Amy Charpentier, "The impact of Service-Learning on Preservice Profesionals' Dispositions Towards Diversity”, Journal of Higher Education Outreach and Engagement 18, 4 (2014): 177-198.

${ }^{15}$ Kelly Lockeman y Lynn E. Pelco, “The Relationship between Service-Learning and Degree Completion”, Michigan Journal of Community Service-Learning 20, 1 (2013): 18-30.

16 Ígor Mella-Núñez, Miguel Ángel Santos-Rego y Xosé Manuel Malheiro-Gutiérrez, "Aprendizaje-servicio y rendimiento académico del alumnado universitario", Revista de Estudios e Investigación en Psicología y Educación, 12 (2015): 35-39, doi: 10.17979/reipe.2015.0.12.569. 
relevancia en la formación de los docentes que van a enseñar ciencias sociales en niveles educativos iniciales. En concreto, fomenta el compromiso, el servicio a la comunidad, la empatía y el voluntariado ${ }^{17}$, de ahí que, tanto en el contexto español como en el anglosajón ${ }^{18}$, se esté introduciendo cada vez más en la educación superior. Las experiencias de aprendizaje servicio abundan cada vez más, pero no dejan de ser algo puntual en el ámbito de la didáctica de las ciencias sociales en España, sobre todo en la etapa de la educación secundaria. Se trata, pues, de una metodología docente no originaria de la enseñanza de la Historia y del patrimonio, pero que presenta gran valor por sí misma si centramos el servicio en el entorno (paisajes, ciudades y monumentos deteriorados o descontextualizados) en que viven personas, grupos y comunidades.

A continuación, se detallan varias experiencias de aprendizaje servicio en el ámbito universitario español que, por razones de espacio, no hacemos extensivo a otros países, en los que tendrían similares motivaciones ${ }^{19}$ (atención a colectivos en riesgo de exclusión social y pobreza).

\section{Algunas experiencias de aprendizaje servicio en la universidad}

Son diversas las experiencias de aprendizaje servicio que se inscriben en la formación inicial de los estudiantes universitarios. En las facultades de educación españolas están creciendo los proyectos de aprendizaje servicio con el objetivo de conectar los contenidos académicos con la realidad profesional y desarrollar la competencia social y ciudadana. Así pues, destacan algunas experiencias llevadas a cabo en el contexto de formación inicial de los futuros educadores.

${ }^{17}$ Timothy K. Stanton, Dwight E. Gilles Jr. y Nadinne I. Cruz, Service-Learning: A Movement's Pioneers Reflect on Its Origins, Practice, and Future (San Francisco: Jossey-Bass Publishers, 1999); Bruce W. Speck y Sherry L. Hoppe (eds.), Service-Learning. History, Theory and Issues (Westport: Praeger, 2004);

${ }^{18}$ Susan J. Deeley, Critical Perspectives on Service-Learning in Higher Education (Londres: Palgrave Macmillan, 2015), 9-29.

${ }^{19}$ Nathalie Ames y Sthephene A. Diepstra, "Using Intergenerational Oral History Service-Learning Projects to Teach Human Behavior Concepts: A Qualitative Analysis”, Educational Gerontology 32, 9 (2006): 721-735, https://doi.org/10.1080/03601270600835447; José Z. Calderón, Race, Poverty and Social Justice. Multidisciplinary Perspectives through Service Learning (Sterling: Stylus, 2007). 


\section{Experiencia de aprendizaje servicio en la Universidad del País Vasco ${ }^{20}$}

Desarrollada en el curso 2013-2014 en la Escuela de Magisterio de Bilbao e inscrita en la asignatura Tecnologías de la Información y la Comunicación de $2 .{ }^{\circ}$ del grado en Educación Social. En ella, estudiantes universitarios y escolares de educación infantil y primaria y sus familias (mayoritariamente de etnia gitana) se implicaron en un proyecto que tenía como objetivo superar la brecha digital, entendida como un elemento configurador de la exclusión socioeducativa. Para cumplir con el objetivo, se realizaron dos talleres en el centro escolar. El primero, de alfabetización digital básica dirigida a familiares y, el segundo, de apoyo para el desarrollo de competencias curriculares TIC. Tras la evaluación, se constató que el alumnado universitario consiguió resultados a nivel académico competencial, cívico, vocacional y profesional, social y personal. Se constató que, tras la experiencia, se había producido un empoderamiento de los padres y las madres ante las tecnologías, se había mejorado su visión del centro y de las actividades que en el mismo desarrollan sus hijos y se había incrementado el interés por participar en actividades.

\section{Experiencia de aprendizaje servicio en la Universidad de La Coruña ${ }^{21}$}

Surge en el curso 2016-2017 en el marco de la asignatura Teoría de la Educación del grado en Educación Primaria. En él han participado ciento treinta estudiantes universitarios y el alumnado y sus maestros de un centro público de educación primaria. Con el proyecto se pretendía promover el pensamiento crítico y reflexivo a través de talleres de filosofía para niños. La evaluación de la experiencia reveló adquisición de conocimientos sobre la filosofía como metodología para el trabajo con escolares.

${ }^{20}$ Begoña Martínez e Isabel Martínez, "El aprendizaje servicio y la formación inicial de profesionales de la educación”, Profesorado: Revista de currículum y formación del profesorado 19, 1 (2015): 245-260.

${ }^{21}$ María-Helena Zapico-Barbeito y María del Camino Pereiro, "Aprendizaje-servicio en la enseñanza universitaria: un proyecto en torno a la Filosofía para niños y niñas”, en Actas del XIV Congreso Internacional de Teoría de la Educación, coords. Ramón Mínguez Vallejos y Eduardo Romero Sánchez (Murcia: Universidad de Murcia, 2017), 695-703. 


\section{Experiencia de aprendizaje servicio en la Universidad de Huelva ${ }^{22}$}

En ella participaron treinta y cuatro estudiantes del grado en Educación Social, treinta y ocho del prado en Psicología, diez del grado en Educación Primaria y catorce del grado en Trabajo Social. Los estudiantes universitarios actuaron como mentores de cuarenta y cuatro alumnos de centros de educación primaria y once alumnos de educación secundaria obligatoria, con el objetivo de prevenir el fracaso escolar. En relación con los estudiantes universitarios, se hallaron efectos positivos articulados con el comportamiento asertivo, se incrementó la motivación extrínseca e intrínseca y se disminuyó la ansiedad ante determinadas situaciones académicas. En cuanto a los alumnos de educación secundaria obligatoria, se observaron mejoras en los resultados escolares y un incremento en el número de asignaturas aprobadas.

\section{Experiencia de aprendizaje servicio en la Universidad de Autónoma de Madrid ${ }^{23}$} Implicó a ciento cuarenta y nueve alumnos de las asignaturas Teoría y Política de la Educación de 1. del grado en Educación Primaria, y Fundamentos teóricos de 1. ${ }^{\circ}$ del grado en Educación Infantil de la Facultad de Formación de Profesorado y Educación. Los alumnos realizaron un mínimo de quince horas de servicio comunitario con personas en situación de desventaja social, exclusión o riesgo de exclusión. Se observaron mejoras en los estudiantes con respecto al compromiso con su propio aprendizaje, al desarrollo de competencias para el ejercicio de la función docente, a la mejora en habilidades de comunicación, al liderazgo y al incremento del compromiso social.

\footnotetext{
${ }^{22}$ Mirian Hervás y Susana Tallón, "El aprendizaje-servicio y mentoría como vía hacia la inclusividad”, en Actas del XIV Congreso Internacional de Teoría de la Educación, coords. Ramón Mínguez Vallejos y Eduardo Romero Sánchez (Murcia: Universidad de Murcia, 2017), 272 281.

${ }^{23}$ Pilar Aramburuzabala y Rocío García, "Aprendizaje-servicio en la formación de maestros comprometidos con la justicia social”, en Universidad y sociedad. Experiencias de aprendizaje servicio en la universidad, coords. Laura Rubio, Enric Prats y Laia Gómez (Barcelona: Universidad de Barcelona, 2013), 14-21.
} 
El aprendizaje servicio como metodología para la enseñanza de la Historia y el patrimonio local

Raimundo A. Rodríguez Pérez - Marta Gutiérrez Sánchez

\section{Experiencia de aprendizaje servicio en la Universidad de Almería ${ }^{24}$}

Se desarrolló en el marco de la asignatura Sociedad, Escuela y Democracia de 1. ․ㅡ de los grados en Educación Infantil y en Educación Primaria. El profesorado de la asignatura estableció contacto con diferentes entidades sociocomunitarias y los estudiantes universitarios diseñaban, desarrollaban y evaluaban sus proyectos de aprendizaje servicio en la entidad que seleccionaban. La evaluación, llevada a cabo en el curso académico 2016-2017, constató que los estudiantes habían obtenido un mayor nivel de dominio de la competencia social y ciudadana y de la capacidad de relacionar la educación con el medio y colaborar con la comunidad.

\section{El aprendizaje servicio para conocer la historia y el patrimonio local}

La novedad que supone esta propuesta didáctica radica en que la mayoría de iniciativas ligadas al aprendizaje servicio versan sobre situaciones de exclusión social. Respecto a la historia local, y el patrimonio vinculado a ella, no se suele plantear este tipo de metodología docente en España, quizá por emanar de la pedagogía y la educación social; si bien existe alguna experiencia previa positiva, por ejemplo, sobre el patrimonio de la ciudad de Cartagena ${ }^{25}$, ubicada también en la región de Murcia. En el ámbito anglosajón es mucho más frecuente también en la enseñanza de la historia y el patrimonio, no en vano el aprendizaje servicio surgió en países de habla inglesa; además, se ha mostrado como una metodología exitosa a la hora de lograr metacognición, altruismo y hacer partícipes a los estudiantes del proceso de enseñanza-aprendizaje ${ }^{26}$. Supone, pues, una forma activa de enseñar en la cual el discente implicado

${ }^{24}$ Victoria Figueredo, Carmen María Hernández y Antonio Martínez, "El aprendizaje-servicio como metodología para favorecer la construcción de la ciudadanía”, en Actas del XIV Congreso Internacional de Teoría de la Educación, coords. Ramón Mínguez Vallejos y Eduardo Romero Sánchez (Murcia: Universidad de Murcia, 2017), 798-803.

25 Álvaro Chaparro Sainz, Salvador Alcaraz García y José Víctor Villalba Gómez, “La formación de maestros a través de un programa de aprendizaje-servicio: un proyecto para enseñar ciencias sociales”, en Investigación en didáctica de las ciencias sociales. Retos, preguntas y líneas de investigación, eds. Ramón Martínez Medina, Roberto García-Moris y Carmen Rosa García Ruiz (Córdoba: Universidad de Córdoba, 2017), 62-71.

${ }^{26}$ Daniel J. Greenberg, “Teaching Global Citizenship, Social Change, and Economic Development in a History Course: A Course Model in Latin American Travel/Service Learning”, History Teacher 41, 3 (2008): 283-304; Alice W. Terry y Thomas Panter, "Students Make Sure the Cherokees are Not Removed... Again: A Study of Service-Learning and Artful Learning in Teaching History", Journal for the Education of the Gifted 34, 1 (2011): 156-176. 
adquiere un protagonismo, inaudito en la enseñanza tradicional basada en lo factual y conceptual (memorización de fechas, datos, personajes).

Conocer el paisaje y el entorno próximos puede ser una herramienta de gran valor educativo, que redunde no sólo en una mejor formación de los estudiantes universitarios (con edades en torno a los 20 años), sino también de los alumnos de educación primaria (6-11 años) y sus familias. La enseñanza de la Historia, en general, y de la historia local ${ }^{27}$, en particular, no suele ser vista como una necesidad por parte de la comunidad educativa, debido al escaso interés que despierta en la sociedad, más allá de estudiosos. Esto denota la persistencia de la Historia como una disciplina erudita, anclada en paradigmas poco atractivos para estudiantes. De ahí la necesidad de sacarla de las aulas para enseñarla in situ, mediante métodos de indagación y estrategias cooperativas ${ }^{28}$ (estudios de caso, salidas escolares, proyectos, aprendizaje basado en problemas). Sin embargo, estas no redundan en un beneficio para la comunidad; algo que sí aporta el aprendizaje servicio por sus orígenes vinculados a la formación de pensamiento crítico y reflexivo en aras de denunciar las injusticias y combatir así las desigualdades sociales y las situaciones de exclusión social.

Por tanto, partiendo de una metodología ajena a la Historia, se pueden extraer pautas dignas de interés que acerquen la historia local como algo atractivo y útil. Es decir, que los estudiantes universitarios y los escolares de educación primaria se sientan partícipes de la recuperación de su pasado,

${ }^{27}$ Julián Casanova Ruiz, "Historia local, historia social y microhistoria”, en La historia local en la España contemporánea: estudios y reflexiones desde Aragón, coords. Ignacio Peiró Martínez y Pedro Víctor Rújula López (Zaragoza: Universidad de Zaragoza, 1999), 17-28.

${ }^{28}$ Araceli Vilarrasa, “Salir del aula. Reapropiarse del contexto”, Íber. Didáctica de las Ciencias Sociales, Geografía e Historia, 36 (2003): 13-25; Montserrat Oller, "Métodos y estrategias para la enseñanza y el aprendizaje de las Ciencias Sociales", en Didáctica del conocimiento del medio social y cultural en la educación primaria, eds. Antoni Santisteban y Joan Pagès (Madrid: Síntesis, 2011), 163-183; Cosme J. Gómez Carrasco y Raimundo A. Rodríguez Pérez, "Aprender a enseñar ciencias sociales con métodos de indagación. Los estudios de caso en la formación del profesorado", Redu: Revista de Docencia Universitaria 12, 2 (2014): 307-325; Ramón Galindo Morales, "Enseñar y aprender Ciencias Sociales en educación primaria. Modelo didáctico y estrategias metodológicas”, en Didáctica de las Ciencias Sociales. Fundamentos, contextos y propuestas, coords. Ángel Liceras Ruiz y Guadalupe Romero Sánchez (Madrid: Pirámide, 2016), 73-94; Mercedes de la Calle, Aprendizaje basado en proyectos (ABP): posibilidades y perspectivas en ciencias sociales, Íber. Didáctica de las Ciencias Sociales, Geografía e Historia, 82 (2016): 7-12.; Martha Cecilia Gutiérrez y Diana Marcela Arana, "La evaluación continua en el ABP”, Íber. Didáctica de las Ciencias Sociales, Geografía e Historia, 88 (2017): 25-29. 
El aprendizaje servicio como metodología para la enseñanza de la Historia y el patrimonio local Raimundo A. Rodríguez Pérez - Marta Gutiérrez Sánchez

convirtiéndose en aprendices de historiadores y arqueólogos. Esto, a su vez, coadyuvará a que adquieran valores cívicos y, a la postre, a generar sociedades más avanzadas.

El propósito de este aprendizaje servicio, que puede implementarse sin grandes dificultades, es convertir la historia local en un recurso educativo, mediante la educación patrimonial. Evitando la tradicional marginación de ese tipo de historia en todos los niveles educativos, dado que suele ser postergada a favor de relatos nacionales y globales ${ }^{29}$. Su uso puede mostrar a estudiantes y docentes la utilidad de la Historia como ciencia social, con unos métodos y fuentes primarias (patrimonio material, en este caso). Dicho recurso educativo deviene en un estímulo para llevar a la práctica la competencia social y cívica, además de otras (lingüística, cultural, tecnológica, aprender a aprender), que capaciten a los futuros maestros y hagan de sus alumnos de educación primaria futuros ciudadanos críticos capaces de respetar y valorar el patrimonio. Este debe entenderse no como edificios o ruinas aisladas, sino como paisajes antropizados, tales como los cascos antiguos de las ciudades y su huerta circundante, con los valores del regadío tradicional.

La cultura del agua, milenaria en la Huerta de Murcia, es la principal seña de identidad de esta comarca. Pero el paso de una agricultura de minifundio a otra tecnificada y exportadora, alejada del entorno de las ciudades, ha hecho que la huerta antigua pierda su función desde la segunda mitad del siglo XX. Ello explica la degradación y desaparición de la red de regadíos (acequias, azarbes, acueductos, molinos, batanes) y el entorno natural asociado a ella, único en tanto que surgido al menos desde el siglo $\mathrm{X}^{30}$ (época islámica), aunque quizá tenga precedentes romanos.

El desprecio y olvido por el patrimonio, por parte de la ciudadanía y sus representantes políticos, ha supuesto un serio daño. Es decir, Murcia-al igual que otras muchas ciudades españolas- pagó un alto precio por el desarrollismo iniciado en la década de 1960, que supuso pasar de una sociedad agraria a otra

${ }^{29}$ Ramón López Facal, "Nacionalismos y europeísmos en los libros de texto: identificación e identidad nacional”, Clío \& Asociados: la historia enseñada, 14 (2010): 9-33; Raimundo A. Rodríguez Pérez y María del Mar Simón García, "La construcción de la narrativa nacional española en los manuales escolares de educación primaria”, Ensayos 29, 1 (2014): 101-113; Raimundo A. Rodríguez Pérez, María del Mar Simón García y Sebastián Molina Puche, "La región de Murcia en los manuales escolares de educación secundaria. Una narrativa a la sombra de España y Europa”, Historia y Memoria de la Educación, 6 (2017): 241-277.

30 José Manzano Martínez, "La agricultura de regadío”, en Guía islámica de la región de Murcia (Murcia: Editora Regional, 1990), 123-139. 
urbana e industrial ${ }^{31}$. En ese período, se perdió buena parte de su casco antiguo (conventos, palacios, construcciones de época musulmana) ${ }^{32}$. Más tarde, le tocó el turno a la zona costera, con una disparatada especulación inmobiliaria, sobre todo en el Mar Menor, laguna salada que arrastra graves problemas medioambientales. La última etapa de esos desmanes urbanísticos afecta a la Huerta de Murcia, que ha perdido la mitad de su superficie cultivada debida, no tanta al crecimiento poblacional, sino a una falta de planificación ordenada.

El urbanismo atroz ha despertado el rechazo de colectivos sociales que promueven recuperar lo mucho que aún queda en los núcleos urbanos y su entorno agrícola. Una riqueza patrimonial amplia y diversa, que va desde yacimientos prehistóricos hasta arqueología industrial, con los restos de fábricas y molinos de apenas un siglo de existencia, pero igualmente arruinados y casi desaparecidos en su totalidad.

Formar maestros de educación primaria en este tipo de iniciativas y contribuir a que colaboren con los precursores de su defensa puede darles una idea de la riqueza de su entorno, que a menudo desconocen e infravaloran, aunque residan y estudien en esa comarca.

La recuperación de la historia local, por medio del patrimonio material en peligro, supone un recurso educativo de primer orden. A su vez, puede impulsar la educación no formal e informal, para el conjunto de la sociedad, comenzando por las familias de los escolares y maestros implicados en la propuesta didáctica sobre el aprendizaje servicio. Por otro lado, recuperar esa herencia cultural y paisajística redundaría en un motor de crecimiento económico, a través de la restauración, puestas en valor y divulgación de la historia. En concreto, el sector de la construcción y el turismo se verían revitalizados, generando ejes de desarrollo cultural y sostenible. Aunque, ante todo, este legado histórico debe ser reconocido por los propios murcianos, que después podrán darlo a conocer a visitantes.

La propuesta didáctica consiste en que los estudiantes de la asignatura Metodología Didáctica para la Enseñanza de las Ciencias Sociales de 3. del grado en Educación Primaria de la Facultad de Educación (Universidad de Murcia) pongan en contacto a colegios de educación primaria con asociaciones

\footnotetext{
${ }^{31}$ Miguel Rodríguez Llopis, Historia de la región de Murcia (Murcia: Editora Regional, 1999), 455-461.

${ }^{32}$ Antonio Martínez-Mena García, "La destrucción del patrimonio arquitectónico y su reflejo en la ciudad de Murcia”, Imafronte, 17 (2003-2004): 127-146.
} 
que promueven la defensa del patrimonio de su entorno próximo ${ }^{33}$. Se trata, pues, de una triple vertiente: universidad, colegios y asociaciones.

En concreto, se han seleccionado tres asociaciones que defienden el patrimonio cultural y natural de la Huerta de Murcia, surgidas en la última década y con amplia repercusión en prensa y redes sociales que han usado para reclamar una mayor atención por un patrimonio harto deteriorado. Dos tienen su sede en el municipio de Murcia (Huermur ${ }^{34}$ y Huerta Viva ${ }^{35}$ ) y una en Alcantarilla $\left(\operatorname{Legado}^{36}\right)$. El éxito de algunas de sus demandas ante las autoridades políticas y judiciales, así como el impacto mediático de sus acciones, ha impulsado la recuperación de ciertos elementos patrimoniales o evitado su desaparición total (puentes, acueductos, acequias, molinos). Por tanto, los maestros en formación deben tomar conciencia del valor que tiene la movilización social para evitar la degradación, aún mayor, de un patrimonio olvidado por el fin de una sociedad agraria que ha dado paso, de forma precipitada, a una sociedad dedicada en su mayor parte a los servicios.

Los maestros en formación deberán analizar el patrimonio en peligro de la comarca de la Huerta de Murcia (sureste de España), concretamente de dos de sus municipios: Murcia y Alcantarilla. A través de la búsqueda en prensa digital (diarios La Verdad y La Opinión de Murcia) verán cuáles son los lugares emblemáticos de ambas localidades y en qué condiciones se encuentran. En pequeños grupos dividirán sus búsquedas en cuatro tipos de elementos patrimoniales: arqueológico, hidráulico, civil y religioso. Una vez elaborado el listado, se seleccionarán aquellos inmuebles o yacimientos más relevantes y a la vez más desprotegidos.

Una vez que los estudiantes han aprendido cuáles son los valores históricoartísticos de la comarca y han analizado su potencial didáctico, la segunda fase es ir a los colegios donde desarrollan las prácticas. La tercera supone que divulguen y colaboren en las acciones de los colectivos citados, dándolos a conocer entre los estudiantes de educación primaria. Para que no quede en

${ }^{33}$ Carlos Ernesto Miranda Carvajal, "El patrimonio local como herramienta educativa”, Revista Internacional de Investigación en Ciencias Sociales 13, 1 (2017): 25-34.

${ }^{34}$ Huermur. Asociación para la Conservación de la Huerta y el Patrimonio de Murcia, 12 de enero de 2018, https://huermur.es/

${ }^{35}$ Huerta Viva. Asociación para la Defensa de la Huerta de Murcia, 12 de enero de 2018, https://www.facebook.com/Murcia-Huerta-Viva-1626105097667138/

${ }^{36}$ Asociación Cultural Legado, 12 de enero de 2018, https://www.facebook.com/Legado-891166100982535/ 
una mera actividad puntual, los maestros en formación deben ir más allá de la acción de voluntariado o de la salida escolar. Así pues, los responsables de las asociaciones irán a los colegios a divulgar sus actuaciones y después mostrarán in situ los principales valores del patrimonio que pretenden proteger.

La duración de esta propuesta sería de dos meses, período que centra las prácticas escolares del segundo cuatrimestre del curso académico, pero debería prepararse durante todo el cuatrimestre anterior. Además, los maestros en formación deberán comprometerse a colaborar con una de las asociaciones una hora semanal, asistiendo a sus reuniones y salidas de campo, a fin de conocer las iniciativas y proyectos, tales como inventario de bienes inmuebles en peligro o reuniones con representantes vecinales.

Los lugares a estudiar de la Huerta de Murcia serían algo más de una treintena, aunque la muestra podría ser más amplia. A continuación, se detalla el listado de bienes y su ubicación:

- Patrimonio arqueológico ${ }^{37}$ :

- Murcia: yacimiento argárico de Puntarrón Chico (Beniaján); martirium romano (La Alberca); basílica paleocristiana (Algezares); arrabal islámico de la Arrixaca (San Esteban); castillo y castillejo de Monteaguado y castillo de Larache (Monteagudo); castillos de Los Garres y Cabezo de Torres.

- Alcantarilla: poblado íbero del Cabezo de la Rueda.

- Patrimonio hidráulico ${ }^{38}$ :

37 José Miguel García Cano, Pasado y presente del patrimonio arqueológico en la región de Murcia (Murcia: Fundación Centro de Estudios Históricos e Investigaciones Locales Región de Murcia, 2006); Antonio Javier Ruiz Medina y José Antonio Sánchez Pravia, Monteagudo: atalaya del tiempo. Una mirada a la identidad cultural del valle de Murcia (Murcia: Natursport, 2014); Julio Navarro Palazón y Pedro Jiménez Castillo, Murcia, la ciudad andalusí que contempló Alfonso X (Murcia: Continental, 2016).

${ }^{38}$ Pedro Díaz Cassou, Ordenanzas y costumbres de la Huerta de Murcia (Madrid: Fortanet, 1889); Manuel Jorge Aragoneses, Museo Etnológico de la Huerta (Alcantarilla, Murcia) (Madrid: Dirección General de Bellas Artes, 1967); Francisco Calvo García-Tornel, Continuidad y cambio en la huerta de Murcia (Murcia: Academia Alfonso X el Sabio, 1982); Julio Muñoz Bravo, Rafael Cortés Gimeno, Miguel Arenillas Parra, Carmen Díaz-Guerra Jaén, “Evolución histórica del complejo hidráulico de la Contraparada”, en Actas del Segundo Congreso Nacional de Historia de la Construcción, ed. Fernando Bores (Coruña: Universidad de La Coruña, 1998), 337-342; Alfredo Morales Gil, Agua y territorio en la región de Murcia (Murcia: Fundación Centro de 
- Murcia: azud de la Contraparada (Javalí Viejo y Javalí Nuevo); rueda y acueducto de La Nora; azud de la Rambla de Zoco (Guadalupe); molinos de la Pólvora (Guadalupe), del Amor (La Albatalía), Oliver (Aljucer) y del Batán (Zarandona).

- Alcantarilla: rueda y acueducto; arcos de la Rambla de las Zorreras; puente de las Pilas.

- Patrimonio civil ${ }^{39}$ :

- Murcia: Cárcel Vieja; Fábrica de Pólvora; Malecón; casas torre del Rincón de Almodóvar (Alquerías), la Cruceta (Rincón de Seca), Falcón (Espinardo) y Guil (Sangonera la Verde).

- Alcantarilla: casa torre de la Voz Negra; estación de ferrocarril; casa modernista Precioso; fábrica de conservas Esteva y Canet.

- Patrimonio religioso ${ }^{40}$ :

Estudios Históricos e Investigaciones Locales Región de Murcia, 2001); Manuel López Campuzano, "Los acueductos de las acequias Alquibla, el Turbedal y la Dava (Alcantarilla): datos arqueológicos sobre la evolución del sistema de regadío rural de la huerta de Murcia durante la alta y baja Edad Media”, Memorias de Arqueología (Murcia: Tres Fronteras, 2001), 636-655; María Martínez Martínez, La cultura del agua en la Murcia medieval (ss. IX-XV) (Murcia: Fundación Centro de Estudios Históricos e Investigaciones Locales Región de Murcia, 2010); Encarna Gil Meseguer, José Antonio López Fernández y José María Gómez Espín, "Regadíos de turbias y secanos asistidos en el sureste de España. El sistema de azud de Guadalupe o presa de derivación de avenidas de la rambla del Zoco (Murcia)", Revista Murciana de Antropología, 22 (2015): 161-176.

${ }^{39}$ Manuel Pérez Sánchez y María Ángeles Gutiérrez García, "El palacio de los marqueses de Espinardo y la villa renacentista”, en El bosque y las villas de recreo en el Renacimiento, coords. Urbano Domínguez Garrido y José Muñoz Domínguez (Salamanca: Junta de Castilla y Léon, 1994), 263-269; Antonio Peñafiel Ramón, El Malecón: su historia (Murcia: Asociación Patrimonio Siglo XXI, 1999); María Griñán Montealegre y Mónica Sánchez López, "El patrimonio artístico, cultural y paisajístico en el casco antiguo de Alcantarilla: un estado de la cuestión”, Imafronte, 16 (2002): 177-194; María Griñán Montealegre, Mónica Sánchez López y María Dolores Palazón Botella, "Propuesta de una ruta de la industria conservera en la región de Murcia”, Imafronte, 19-20 (2007-2008): 105-118; Francisco José Medina Albaladejo, "La Prisión Provincial de Murcia: aproximación arquitectónica, histórica y cultural”, Anales de Historia Contemporánea, 25 (2009): 407-427.

${ }^{40}$ Cristina Gutiérrez-Cortines Corral, La Iglesia y Colegio de San Esteban de Murcia (Murcia: Diputación Provincial, 1976); Juan José Franco Manzano, Arte y tradición en la Huerta de Murcia (Murcia: Editora Regional, 2001); Mariano C. Pelegrín Garrido, Las ermitas de Murcia, su huerta y su campo (Murcia: Ayuntamiento de Murcia, 2010). 
- Murcia: iglesia de San Esteban; ermita de los Pasos de Santiago.

- Alcantarilla: ermita de la Voz Negra.

Los cuatro tipos de elementos patrimoniales de la Huerta de Murcia se han categorizado a partir de su función primigenia, aunque Cuenca, Estepa y Martín aluden a otro tipo de clasificación del patrimonio, con cuatro vertientes: histórico-artístico, natural, etnográfico y tecnológico ${ }^{41}$. El primero de ellos incluiría tres de las categorías aquí empleadas (arqueológico, civil y religioso), en tanto que el hidráulico sería a la vez etnográfico y tecnológico. El natural sería el entorno de huerta que engloba la comarca homónima de Murcia, es decir, el regadío tradicional, derivado de una agrociudad y las localidades que le rodean (varias decenas de pedanías y tres municipios: Alcantarilla, Beniel y Santomera).

\section{Diseño de la propuesta de aprendizaje servicio sobre historia local}

Para garantizar que las propuestas de esta naturaleza supongan la formación ciudadana, por un lado, y la adquisición de contenidos curriculares, por otro, es fundamental que se atienda a una lógica de planificación. En el trabajo arriba mencionado (Ministerio de Educación de Argentina ${ }^{42}$ ) se organizan, de manera muy clara, los pasos a seguir para el diseño de este tipo de proyectos. Insistiendo inicialmente en que la propia elaboración del proyecto constituye en sí misma una experiencia de aprendizaje que permite, asimismo, que el alumnado se responsabilice y comprometa durante todo el proceso al otorgarle un papel activo, se distinguen las siguientes etapas de planificación:

\subsection{Motivación}

Se trata de implicar y responsabilizar lo máximo posible a todos los actores que, de manera directa e indirecta, participarán en el proyecto de servicio comunitario. Supone tener en cuenta dos cuestiones: por un lado, la motivación personal e institucional para el desarrollo del proyecto y, por otro, el conocimiento y comprensión del concepto de aprendizaje servicio.

En esta primera fase se presentaría la propuesta a los maestros en formación de la Universidad de Murcia, así como a los colegios (al menos diez durante el

\footnotetext{
${ }^{41}$ José María Cuenca López, Jesús Estepa Giménez y Myriam José Martín Cáceres, "Patrimonio, educación, identidad y ciudadanía. Profesorado y libros de texto en la enseñanza obligatoria", Revista de Educación, 375 (2017): 146, Doi: 10.4438/1988-592X-RE-2016-375-338.

${ }^{42}$ Ministerio de Educación de Argentina. Itinerario y herramientas, 33-54.
} 
primer curso en el que se desarrollase el proyecto) de educación primaria en los que van a hacer las prácticas, preferentemente de los municipios de Murcia y Alcantarilla, zona en la que desempeñan su actividad las tres asociaciones citadas. Se establecería un calendario diferenciado en dos partes: primer cuatrimestre, con actuaciones en la Universidad de Murcia, dentro de la asignatura de referencia y colaboración con los colectivos pro Huerta de Murcia; segundo cuatrimestre, actuación conjunta de colegios y asociaciones, con la supervisión de los docentes universitarios que coordinan la actividad.

\subsection{Diagnóstico}

Supone identificar la necesidad o el problema de la comunidad sobre la que se va a intervenir. Es importante que todos los actores se impliquen en dicha identificación para asegurar la efectividad del servicio comunitario. Asimismo, en esta fase es importante analizar la posibilidad de respuesta que tiene la institución promotora del proyecto en función de su identidad y de acuerdo con los recursos, prioridades y tiempos disponibles.

En esta fase habría que solicitar los permisos pertinentes a la Facultad de Educación (Universidad de Murcia), concejalías de educación de los ayuntamientos de Murcia y Alcantarilla, y Consejería de Educación del gobierno autonómico de la región de Murcia. Dado que se trabajará con estudiantes menores de edad, debe seguirse un protocolo de actuación, además de mostrar el calendario y acciones propuestas, a fin de que los respectivos organismos (universitario, municipal y autonómico) sean conocedores y copartícipes del proyecto de aprendizaje servicio. No sería necesario pedir ayuda financiera, salvo para algún desplazamiento de los estudiantes a lugares cercanos de su propio municipio, con cortos trayectos en autocar. Los estudiantes universitarios y los maestros que tutelen sus prácticas en los colegios necesitarían informar de esas actividades, pudiendo así integrarlas en su programación docente.

\subsection{Diseño y planificación}

En esta fase se trata de articular todos los elementos clásicos de diseño de cualquier proyecto de intervención socioeducativa, aunque atendiendo a algunas particularidades que son propias del aprendizaje servicio. En este sentido, se tendrán que definir los objetivos - diferenciando entre objetivos de aprendizaje y objetivos de acción solidaria-, fijar los destinatarios, las actividades, la temporalización, los responsables y los recursos. Señalar aquí, 
por ser la piedra angular de este tipo de proyectos, la necesidad de articular curricularmente el proyecto identificando con claridad qué asignaturas o áreas y contenidos concretos desarrollarán los estudiantes con la acción solidaria.

Para acometer este proyecto los estudiantes universitarios diseñarán actividades para sus prácticas escolares del segundo cuatrimestre del curso, durante el cuatrimestre previo, en el marco de la asignatura Metodología Didáctica para la Enseñanza de las Ciencias Sociales (3.ํ de Educación Primaria). En pequeños grupos (4-6 estudiantes) se analizarán cada uno de los emblemas patrimoniales en peligro. Dichos inmuebles serán repartidos por sorteo, a partir de la clasificación antes citada. Así pues, un grupo hará solamente propuestas de patrimonio hidráulico, mientras que los demás se ocuparán del resto de tipologías (arqueológica, civil y religiosa).

En las clases prácticas se analizarán qué tipo de medidas promueven las tres asociaciones que velan por la defensa del patrimonio de la Huerta de Murcia, confiriendo así coherencia a las propuestas. Miembros de dichas asociaciones serán invitados al aula para explicar sus líneas maestras y, después, se harán sesiones de trabajo de campo con ellos. Los estudiantes colaborarán en acciones de prospección arqueológica, fotografía, búsqueda de documentación en prensa y archivos digitales, análisis de la legislación sobre patrimonio y asistencia a reuniones de trabajo.

Esas actividades serán expuestas en pequeños grupos y coevaluadas en la parte práctica de la asignatura. Los demás compañeros y el docente corregirán - con el mismo peso en la calificación final, mediante una rúbrica analítica-las propuestas elaboradas por escrito y su capacidad para exponerlas de forma oral. El debate sobre lo expuesto, sus logros y carencias, dará pie a un formato de actividad lista para aplicarse en los colegios de educación primaria.

\subsection{Ejecución}

Supone la puesta en práctica de lo planificado. En esta etapa es cuando se desarrollan los contenidos curriculares previstos en la fase de diseño por lo que se podrán combinar actividades de práctica solidaria con actividades formativas de tipo curricular, necesarias para llevar a cabo las acciones de servicio comunitario.

Esta fase es la más relevante. Se dará en el segundo cuatrimestre del curso, concretamente durante los meses de febrero y marzo, cuando los estudiantes universitarios hacen prácticas en los diez colegios de enseñanza primaria seleccionados. Esto supondría, al menos, otros tantos docentes en formación 
implicados, es decir, diez. Cada uno sería maestro en prácticas de un grupoclase de educación primaria, preferentemente de segundo tramo de dicha etapa educativa (4., $5 .^{\circ}$ y $6 .{ }^{\circ}$ curso: nueve, diez y once años de edad, respectivamente), que es cuando se abordan los contenidos disciplinares de Historia y patrimonio desde la prehistoria hasta la época actual, según el currículum vigente (Ley Orgánica para la Mejora de la Calidad Educativa, Boletín Oficial de la Región de Murcia, Decreto 198/2014). Cada grupo-clase cuenta con una media de 25 estudiantes de enseñanza primaria. Por tanto, la muestra con estudiantes de nueve a once años rondaría los 250 que, como pilotaje, sería relevante para poner a prueba las ventajas y desventajas del proyecto, de cara a siguientes cursos académicos.

Tiene una misión doble esta fase. Por un lado, aplicar al menos una de las actividades puntuales sobre historia local, diseñada en el cuatrimestre anterior, y evaluarla para conocer la respuesta de los alumnos de educación primaria. Para ello, se contará con la ayuda de los maestros-tutores de prácticas. Por otro lado, se darán a conocer en el colegio las acciones de las tres asociaciones con las que se colabora. Charlas, realización de trabajos grupales sobre la historia de la Huerta de Murcia, exposición de los mismos en el centro escolar. Pero, sobre todo, habrá que salir del aula para estudiar in situ algunos de los bienes en peligro de su localidad y compararlos con otros recuperados (o en vías de serlo). Las familias pueden sumarse a este tipo de iniciativas, sobre todo si se emprenden como complemento formativo en horario extraescolar y dado la cercanía de los lugares a estudiar.

\subsection{Cierre}

Es aquí cuando se valoran los resultados en cuanto a los aprendizajes curriculares adquiridos y los objetivos que se pretendían conseguir con los destinatarios del servicio solidario. Esta valoración será más enriquecedora si se tienen en cuenta las percepciones de los estudiantes, los destinatarios del proyecto y todos los profesionales implicados. Esta fase es fundamental para recoger información que mejore el proyecto de cara a futuras intervenciones. Además, permitirá difundir la experiencia, cuestión que podrá redundar en una valoración positiva por parte de la comunidad y, por ende, en la implicación de otros actores e instituciones.

Esta parte final requiere un proceso de metaevaluación del aprendizaje servicio para poner de relieve ventajas e inconvenientes del proceso. La parte docente se evaluará mediante el análisis de los trabajos realizados, tanto por 
maestros en formación como por alumnos de enseñanza primaria, por medio de rúbricas ${ }^{43}$ que evalúen el logro de conceptos de pensamiento histórico: relevancia histórica, fuentes, cambio y continuidad, causas y consecuencias, perspectiva histórica, dimensión ética de la historia ${ }^{44}$.

Junto a esa evaluación de corte cuantitativo, cabría una evaluación complementaria de índole cualitativa. Se basaría en los portafolios de los maestros en formación, a lo largo de todo el curso académico, con sus reflexiones acerca de la metodología aprendizaje servicio: su primera fase, a modo de ensayo en las aulas universitarias, la colaboración con entidades cívicas; y su puesta en práctica en colegios de enseñanza primaria. Esta herramienta resulta esencial, según Deeley ${ }^{45}$, una de las mayores especialistas en aprendizaje servicio, para su positiva repercusión en la formación de estudiantes universitarios. Dicha autora hace especial hincapié en la necesidad de valorar la autocrítica y cómo se han afrontado las dificultades a lo largo de todo el proceso, que deben reflejarse en los citados portafolios.

En cuanto a la parte de servicio a la comunidad, se basará en las respuestas de los cuestionarios dadas por maestros en formación, alumnos de educación primaria, familiares de los mismos, maestros-tutores y miembros de las asociaciones colaboradoras. El cuestionario debe recoger qué se conoce-antes y después del proyecto educativo-sobre los siguientes aspectos: la historia local; el valor otorgado al patrimonio como fuente primaria de la historia; edificios y lugares; necesidad de recuperar el legado cultural y el paisaje circundante.

La divulgación correría a cargo de la Facultad de Educación y las administraciones implicadas (ayuntamientos y comunidad autónoma) que, a través de la prensa y sus respectivas páginas web, darían a conocer el proceso y los resultados obtenidos. En caso contrario, no habría repercusión y quedaría en una iniciativa aislada.

\section{Conclusiones}

El aprendizaje servicio puede contribuir a mejorar la enseñanza de la Historia, tanto en maestros en formación como en alumnos de educación primaria. Aquellos pueden aprender a usar herramientas metodológicas propias de

43 Pedro Miralles Martínez y José Díaz-Serrano, "El proceso evaluativo en la enseñanza de la historia”, Andamio 3, 2 (2016): 13-34.

${ }^{44}$ Peter Seixas y Tom Morton, The Big Six Historical Thinking Concepts (Toronto: Nelson, 2013).

${ }^{45}$ Deeley, Critical Perspectives, 81-102. 
historiadores e investigadores sociales (arqueólogos, historiadores del arte, geógrafos, antropólogos) al conocer las iniciativas de las asociaciones que más están despertando las conciencias sobre el deterioro del acervo común de la Huerta de Murcia y sus dos núcleos urbanos más importantes. Es decir, experimentar la utilidad y complejidad de la enseñanza de las ciencias sociales, a través del estudio de la historia y el patrimonio local en peligro. En cuanto a los escolares de educación primaria, podrán estudiar la Historia de forma amena, alejada del tradicional currículum culturalista, apoyado en manual, lección magistral y examen memorístico escrito, una trilogía que les desmotiva. Se debe salir del aula y convertir el barrio o la localidad en un inmenso taller a cielo abierto que, si se explica con sencillez, puede arrojar mucha luz sobre nuestro pasado colectivo: tecnologías, transportes, comercio, vida cotidiana, religiosidad, ocio, alimentación.

El aprendizaje servicio puede ayudar a sacar la enseñanza de la Historia de su letargo tradicional y conectar, no sólo la universidad con los centros educativos, sino con entidades sociales que están consiguiendo más que los poderes fácticos y mediáticos, poco proclives a la cultura de base. El hartazgo por la dejadez de las instituciones respecto a la historia y el patrimonio local puede combatirse con educación, desde los niveles escolares iniciales. Ya que los decretos curriculares de cualquier etapa impiden acercarse a lo local con asiduidad, deben ser docentes y discentes quienes elaboren proyectos de aprendizaje propios que impliquen a la sociedad y a las familias, redundando en la adquisición de valores de respeto y empatía por el legado de los antepasados.

Con todo, esta propuesta no es sino un adelanto, cuya utilidad real sólo podrá vislumbrarse cuando se lleve a la práctica y sea evaluada, con sus pros y sus contras. No obstante, puede intuirse que arrojaría datos relevantes, dada la relativa amplitud de la muestra (varios centenares de estudiantes de educación primaria, decenas de maestros en formación y docentes) y la interconexión entre instituciones educativas (colegios y universidad), a menudo separadas, pero cuya colaboración es frecuente y fluida de forma anual por medio de las prácticas curriculares de los estudiantes universitarios en las aulas de educación primaria. A su vez, implicar a un tercer agente, los colectivos conservacionistas, supone dotar a la propuesta didáctica de aprendizaje servicio de una utilidad más allá de las aulas y del sistema educativo, llegando al contexto social y a las localidades que albergan monumentos y paisajes en trance de desaparición.

La Huerta de Murcia, a pesar de ser la comarca que circunda - y conformael séptimo municipio más poblado de España, sufre un acelerado proceso de 
deterioro por la pérdida de su tradicional función de sustento agrícola de las familias. Ahora casi nadie vive del sector primario en esta comarca, pero una sociedad que ignora sus raíces está destinada a perder su propia identidad.

\section{Referencias bibliográficas}

\section{Fuentes secundarias}

Ames, Nathalie y Diepstra, Sthephene A. "Using Intergenerational Oral History Service-Learning Projects to Teach Human Behavior Concepts: A Qualitative Analysis". Educational Gerontology 32, 9 (2006): 721-735, DOI: https:/doi. org $/ 10.1080 / 03601270600835447$

Aramburuzabala, Pilar y Rocío García. "Aprendizaje-servicio en la formación de maestros comprometidos con la justicia social", en Universidad y sociedad. Experiencias de aprendizaje servicio en la universidad, coordinado por Laura Rubio, Enric Prats y Laia Gómez, 14-21. Barcelona: Universidad de Barcelona, 2013.

Bauman, Zygmunt. Vida líquida. Barcelona: Paidós, 2006.

Byun-Chul, Han. La expulsión de lo distinto. Barcelona: Herder, 2017.

Calderón, José Z. Race,Poverty and Social Justice. Multidisciplinary Perspectives through Service Learning. Sterling: Stylus, 2007.

Calvo García-Tornel, Francisco. Continuidad y cambio en la huerta de Murcia. Murcia: Academia Alfonso X el Sabio, 1982.

Casanova Ruiz, Julián. "Historia local, historia social y microhistoria”, en La historia local en la España contemporánea: estudios y reflexiones desde Aragón, coordinado por Ignacio Peiró Martínezy Pedro Víctor Rújula López, 17-28. Zaragoza: Universidad de Zaragoza, 1999.

Chaparro Sainz, Álvaro, Salvador Alcaraz García y José Víctor Villalba Gómez. "La formación de maestros a través de un programa de aprendizaje-servicio: un proyecto para enseñar ciencias sociales", en Investigación en didáctica de las ciencias sociales. Retos, preguntas y líneas de investigación, editado por Ramón Martínez Medina, Roberto García-Moris y Carmen Rosa García Ruiz, 62-71. Córdoba: Universidad de Córdoba, 2017.

Composto, Claudia y Mina Lorena Navarro. "Estados, transnacionales extractivas y comunidades movilizadas: dominación y resistencias en torno de la minería a gran escala en América Latina”. Revista Theomai ,25, 1 (2012): 58-78.

Cuenca López, José María, Jesús Estepa Giménez y Myriam José Martín Cáceres. "Patrimonio, educación, identidad y ciudadanía. Profesorado y libros de texto en la enseñanza obligatoria”. Revista de Educación, 375 (2017): 136-159, doi: 10.4438/1988-592X-RE-2016-375-338

De la Calle, Mercedes. Aprendizaje basado en proyectos (ABP): posibilidades y perspectivas en ciencias sociales. Íber. Didáctica de las Ciencias Sociales, Geografía e Historia, 82 (2016): 7-12. 
El aprendizaje servicio como metodología para la enseñanza de la Historia y el patrimonio local

Raimundo A. Rodríguez Pérez - Marta Gutiérrez Sánchez

Deeley, Susan J. Critical Perspectives on Service-Learning in Higher Education. Londres: Palgrave Macmillan, 2015.

Díaz Cassou, Pedro. Ordenanzas y costumbres de la Huerta de Murcia. Madrid: Fortanet, 1889.

Figueredo, Victoria, Carmen María Hernández y Antonio Martínez. "El Aprendizaje-Servicio como metodología para favorecer la construcción de la ciudadanía”, en Actas del XIV Congreso Internacional de Teoría de la Educación, coordinado por Ramón Mínguez Vallejos y Eduardo Romero Sánchez, 798-803. Murcia: Universidad de Murcia, 2017.

Franco Manzano, Juan José. Arte y tradición en la Huerta de Murcia. Murcia: Editora Regional, 2001.

Furco, Andrew. "Impacto de los proyectos de aprendizaje-servicio", en Actas del séptimo Seminario Internacional Aprendizaje y Servicio Solidario,19-26. Buenos Aires, 2004.

Galindo Morales, Ramón. "Enseñar y aprender Ciencias Sociales en educación primaria. Modelo didáctico y estrategias metodológicas", en Didáctica de las Ciencias Sociales. Fundamentos, contextos y propuestas, coordinado por Ángel Liceras Ruiz y Guadalupe Romero Sánchez, 73-94. Madrid: Pirámide, 2016.

García Cano, José Miguel. Pasado y presente del patrimonio arqueológico en la región de Murcia. Murcia: Fundación Centro de Estudios Históricos e Investigaciones Locales Región de Murcia, 2006.

Gil Meseguer, Encarnación, José Antonio López Fernández y José María Gómez Espín. "Regadíos de turbias y secanos asistidos en el sureste de España. El sistema de azud de Guadalupe o presa de derivación de avenidas de la rambla del Zoco (Murcia)”. Revista Murciana de Antropología, 22 (2015): 161-176.

Glazier, Jocelyn, Harriet Able y Amy Charpentier. “The impact of Service-Learning on Preservice Profesionals' Dispositions Towards Diversity”. Journal of Higher Education Outreach and Engagement 18, 4 (2014): 177-198.

Gómez Carrasco, Cosme J. y Raimundo A. Rodríguez Pérez. “Aprender a enseñar ciencias sociales con métodos de indagación. Los estudios de caso en la formación del profesorado”. Redu: Revista de Docencia Universitaria, 12, 2 (2014): 307-325.

Greenberg, Daniel J. "Teaching Global Citizenship, Social Change, and Economic Development in a History Course: A Course Model in Latin American Travel/ Service Learning”. History Teacher 41, 3 (2008): 283-304.

Griñán Montealegre, María y Mónica Sánchez López. "El patrimonio artístico, cultural y paisajístico en el casco antiguo de Alcantarilla: un estado de la cuestión”. Imafronte, 16 (2002): 177-194.

Griñán Montealegre, María, Mónica Sánchez López y María Dolores Palazón Botella. "Propuesta de una ruta de la industria conservera en la región de Murcia". Imafronte, 19-20 (2007-2008): 105-118.

Gutiérrez, Martha Cecilia y Diana Marcela Arana. "La evaluación continua en el ABP”. Iber. Didáctica de las Ciencias Sociales, Geografía e Historia, 88 (2017): 25-29. 
Gutiérrez-Cortines Corral, Cristina. La Iglesia y Colegio de San Esteban de Murcia. Murcia: Diputación Provincial, 1976.

Hervás, Mirian y Susana Tallón. "El aprendizaje-servicio y mentoría como vía hacia la inclusividad”, en Actas del XIV Congreso Internacional de Teoría de la Educación, coordinado por Ramón Mínguez Vallejos y Eduardo Romero Sánchez, 272-281. Murcia: Universidad de Murcia, 2017.

Houtart, François. “De los bienes comunes al bien común de la humanidad”. El Ágora USB 14,1 (2014): 259-293.

Huerta Viva. Asociación para la Defensa de la Huerta de Murcia. [En línea], https:// www.facebook.com/Murcia-Huerta-Viva-1626105097667138/ Consultado 12 de enero, 2018.

Jorge Aragoneses, Manuel. Museo Etnológico de la Huerta (Alcantarilla. Murcia). Madrid: Dirección General de Bellas Artes, 1967.

Lockeman, Kelly y Lynn E. Pelco. "The Relationship between Service-Learning and Degree Completion”. Michigan Journal of Community Service-Learning 20, 1 (2013): 18-30.

López Campuzano, Manuel. "Los acueductos de las acequias Alquibla, el Turbedal y la Dava (Alcantarilla): datos arqueológicos sobre la evolución del sistema de regadío rural de la huerta de Murcia durante la alta y baja Edad Media”, en Memorias de Arqueología, 636-655. Murcia: Tres Fronteras, 2001.

López Facal, Ramón. "Nacionalismos y europeísmos en los libros de texto: identificación e identidad nacional”. Clío \& Asociados: la historia enseñada, 14 (2010): 9-33.

Maiso, Jordi. "Sobre la producción y reproducción social de la frialdad", en Las víctimas como precio necesario, editado por José Antonio Zamora, Manuel Reyes Mate y Jordi Maiso, 51-69. Madrid: Trotta, 2016.

Manzanera, María. Murcia huertana. Murcia: Fundación Cajamurcia, 2014.

Manzano Martínez, José. "La agricultura de regadío”. En Guía islámica de la región de Murcia, 123-139. Murcia: Editora Regional, 1990.

Martínez Martínez, María. La cultura del agua en la Murcia medieval (ss. IX-XV). Murcia: Fundación Centro de Estudios Históricos e Investigaciones Locales Región de Murcia, 2010.

Martínez, Begoña e Isabel Martínez. "El aprendizaje servicio y la formación inicial de profesionales de la educación”. Profesorado: Revista de currículum y formación del profesorado 19, 1 (2015): 245-260.

Martínez, Miquel. "Aprendizaje servicio y construcción de ciudadanía activa en la universidad: la dimensión social y cívica de los aprendizajes académicos”, En Aprendizaje servicio y responsabilidad social de las universidades, editado por Miquel Martínez, 11-26. Barcelona: Octaedro, 2008.

Martínez-Mena García, Antonio. "La destrucción del patrimonio arquitectónico y su reflejo en la ciudad de Murcia”. Imafronte, 17 (2003-2004): 127-146.

Medina Albaladejo, Francisco José. "La Prisión Provincial de Murcia: aproximación arquitectónica, histórica y cultural”. Anales de Historia Contemporánea, 25 (2009): 407-427. 
Mella-Núñez, Íor, Miguel Ángel Santos-Rego y Xosé Manuel Malheiro-Gutiérrez. "Aprendizaje-servicio y rendimiento académico del alumnado universitario". Revista de Estudios e Investigación en Psicología y Educación, 12 (2015): 35-39, doi: 10.17979/reipe.2015.0.12.569.

Ministerio de Educación de Argentina. Itinerario y herramientas para desarrollar un proyecto de aprendizaje-servicio. Buenos Aires: Ministerio de Educación de la Nación, 2012.

Miralles Martínez, Pedro y José Díaz-Serrano. “El proceso evaluativo en la enseñanza de la historia". Andamio 3, 2 (2016): 13-34.

Miranda Carvajal, Carlos Ernesto. "El patrimonio local como herramienta educativa”. Revista Internacional de Investigación en Ciencias Sociales 13, 1 (2017): 25-34.

Morales Gil, Alfredo. Agua y territorio en la región de Murcia. Murcia: Fundación Centro de Estudios Históricos e Investigaciones Locales Región de Murcia, 2001.

Muñoz Bravo, Julio, Rafael Cortés Gimeno, Miguel Arenillas Parra y Carmen Díaz-Guerra Jaén. "Evolución histórica del complejo hidráulico de la Contraparada”, en Actas del Segundo Congreso Nacional de Historia de la construcción, editado por Fernando Bores, 337-342. La Coruña: Universidad de La Coruña, 1998.

Navarro Palazón, Julio y Pedro Jiménez Castillo. Murcia, la ciudad andalusí que contempló Alfonso X. Murcia: Continental, 2016.

Oller, Montserrat. "Métodos y estrategias para la enseñanza y el aprendizaje de las Ciencias Sociales", en Didáctica del conocimiento del medio social y cultural en la educación primaria, editado por Antoni Santisteban y Joan Pagès, 163-183. Madrid: Síntesis, 2011.

Pelegrín Garrido, Mariano C. Las ermitas de Murcia, su huerta y su campo. Murcia: Ayuntamiento de Murcia, 2010.

Peñafiel Ramón, Antonio. El Malecón: su historia. Murcia: Asociación Patrimonio Siglo XXI, 1999.

Pérez Sánchez, Manuel y María Ángeles Gutiérrez García. "El palacio de los marqueses de Espinardo y la villa renacentista", en El bosque y las villas de recreo en el Renacimiento, coordinado por Urbano Domínguez Garrido y José Muñoz Domínguez, 263-269. Salamanca: Junta de Castilla y Léon, 1994.

Rodríguez Llopis, Miguel. Historia de la región de Murcia. Murcia: Editora Regional, 1999.

Rodríguez Pérez, Raimundo A. y María del Mar Simón García. “La construcción de la narrativa nacional española en los manuales escolares de educación primaria". Ensayos 29, 1 (2014): 101-113.

Rodríguez Pérez, Raimundo A., María del Mar Simón García y Sebastián Molina Puche. "La región de Murcia en los manuales escolares de educación secundaria. Una narrativa a la sombra de España y Europa”. Historia y Memoria de la Educación, 6 (2017): 241-277.

Ruiz Medina, Antonio Javier y José Antonio Sánchez Pravia. Monteagudo: atalaya del tiempo. Una mirada a la identidad cultural del valle de Murcia. Murcia: Natursport. 2014. 
Seixas, Peter y Tom Morton. The Big Six Historical Thinking Concepts. Toronto: Nelson, 2013.

Speck, Bruce W. y Sherry L. Hoppe (eds.). Service-Learning. History, Theory and Issues. Westport: Praeger, 2004.

Stanton, Timothy K., Dwigth E. Gilles Jr. y Nadinne I. Cruz. Service-Learning: A Movement's Pioneers Reflect on Its Origins, Practice, and Future. San Francisco: Jossey-Bass Publishers, 1999.

Terry, Alice W. y Thomas Panter. "Students Make Sure the Cherokees are Not Removed... Again: A Study of Service-Learning and Artful Learning in Teaching History". Journal for the Education of the Gifted 34, 1 (2011): 156-176.

Torío, Susana. "Familia, Escuela y Sociedad". Aula Abierta, 83 (2004): 35-52.

Vaknin, Lauren Weiner y Marilee J. Bresciani. "Implementing Quality Service-Learning Programs in Community Colleges”. Journal of Research and Practice 37, 12 (2013): 979-989, doi:10.108 0/10668926.2010.515515

Vilarrasa, Araceli. “Salir del aula. Reapropiarse del contexto”. Íber. Didáctica de las Ciencias Sociales, Geografía e Historia, 36 (2003): 13-25.

Zapico-Barbeito, María-Helena y María del Camino Pereiro. "Aprendizaje-servicio en la enseñanza universitaria: un proyecto en torno a la Filosofía para niños y niñas”, en Actas del XIV Congreso Internacional de Teoría de la Educación, coordinado por Ramón Mínguez Vallejos y Eduardo Romero Sánchez, 695-703. Murcia: Universidad de Murcia, 2017. 
